\title{
Use of new anticoagulants for the treatment of venous thromboembolism in the context of the results of the XALIA study - clinical trials and routine clinical practice
}

\author{
Tomasz Urbanek
}

Department of General Surgery, Vascular Surgery, Angiology and Phlebology, Medical University of Silesia, Katowice, Poland

\begin{abstract}
The introduction of new non-vitamin K antagonist oral anticoagulants (NOAC) has had a significant impact on the treatment regimen of patients with venous thromboembolism. Phase 3 studies with the use of this group of drugs, including both direct factor II inhibitors and direct factor Xa inhibitors, have shown that the safety and effectiveness of this type of treatment are similar to vitamin K antagonists that have been used for a long time. Due to the eligibility of randomly selected patients that meet strict and specific inclusion criteria for pre-marketing studies, studies based on the assessment of the effectiveness and clinical safety of the above-mentioned drugs in the routine clinical practice are becoming extremely important. XALIA, the first international, multicenter, prospective clinical study on the use of the direct coagulation inhibitor — rivaroxaban in the routine clinical practice is an example of these types of studies. The aim of the study was to compare the results of the anticoagulant therapy in patients with deep vein thrombosis and patients with combined deep vein thrombosis and pulmonary embolism using rivaroxaban and standard anticoagulation regimen in the routine clinical practice. The results of the XALIA study confirm the findings of previous prospective, randomized studies in the population characterized by a broad spectrum of patients, indicating the safety and effectiveness of rivaroxaban in the treatment of venous thromboembolism in the routine clinical practice. The incidence of major bleeding complications in the group of patients receiving rivaroxaban was $0.7 \%$ compared to $0.8 \%$ of patients treated using standard anticoagulants. The proportion of recurrence of venous thromboembolism was low and similar to the results obtained in the EINSTEIN DVT study [XALIA - 1.4\% vs. EINSTEIN DVT - 2.1\%]. No cases of fatal bleeding were reported in the XALIA study and the calculated proportion of major bleeding complications in patients taking rivaroxaban was 1.2 per year, the proportion of recurrence and death from any cause was $2.4 \%$ and $0.8 \%$, respectively. The obtained results confirm previous observations suggesting a low risk of recurrence as well as a high safety of the anticoagulant therapy in patients with deep vein thrombosis using rivaroxaban - this time in the study related to the routine clinical practice.
\end{abstract}

Key words: venous thromboembolism, oral anticoagulants, rivaroxaban, complications

Acta Angiol 2016; 22, I: 28-36

The introduction of new oral direct anticoagulants has had a significant impact on the approach to the current treatment regimen of patients with venous thromboembolism in recent years [I-5]. The proven and widely used treatment method based on using heparins and vitamin $\mathrm{K}$ antagonist oral anticoagulants is increasingly being replaced by the treatment based on the use of new molecules registered in this indica- 
tion $[1-3,6]$. At present, available registries of both factor $X a$ inhibitors and thrombin inhibitors allow for the treatment of deep vein thrombosis and pulmonary embolism (in accordance with SmPC) as well as the prevention of recurrent deep vein thrombosis and pulmonary embolism in adults $[3,6-10]$. There are also indications related to the use of these drugs in the prevention of venous thromboembolism; nevertheless, the latter are limited to hip and knee replacement [6-10]. New anticoagulants may be used in conjunction with the initial treatment with parenteral, short-acting anticoagulants (factor Xa inhibitors, thrombin inhibitors) as well as in the monotherapy (factor Xa inhibitors) [6-10].

The increasing use of new treatment methods makes it necessary to verify the results of pre-marketing randomized, clinical trials (phase 3 trials) in prospective studies related to the routine clinical practice in the scope of the safety and effectiveness of the suggested treatment method.

The populations of patients eligible for phase 3 trials are usually subject to strictly defined inclusion and exclusion criteria and they are not always comparable to a wide spectrum of populations of patients treated within the so-called "routine clinical practice". Will the positive results of the treatment of patients with venous thromboembolism using new molecules documented in the phase 3 trials, which are at least similar to the results of current treatment methods, be therefore reflected in the everyday and increasing use in the clinical practice of different specialists?

According to the results of the majority of previous phase 3 trials, the use of drugs from the groups of direct coagulation inhibitors met the criteria of non-inferiority studies in relation to the use of the current anticoagulant therapy [I-14]. In the EINSTEIN DVT study, the proportion of patients with major bleeding complications in the groups taking rivaroxaban was $0.8 \%$ and in the group treated with a standard anticoagulant therapy - $1.2 \%$ with a similar incidence of major and clinically significant bleeding complications $(8.1 \%$ vs. $8.1 \%)$ during treatment [II]. The primary endpoint including the recurrence of symptomatic venous thromboembolism defined as the occurrence of deep vein thrombosis and (fatal or non-fatal) pulmonary embolism occurred in $2.1 \%$ of patients treated with rivaroxaban and $3 \%$ of patients treated with the standard therapy, respectively [I I]. In the study related to the treatment of pulmonary embolism (EINSTEIN $\mathrm{PE})$, the proportion of major bleeding complications was $1.1 \%$ in case of rivaroxaban and $2.2 \%$ in case of the standard therapy, respectively, while the results in the scope of the effectiveness were similar [12]. In the studies with the use of dabigatran (RECOVER II), $1.2 \%$ of cases of major bleeding complications were reported in patients treated with dabigatran and $1.7 \%$ in patients treated with the standard protocol [13]. At the same time, the outcome of the study (the recurrence of symptomatic venous thromboembolism or death due to this disease) was reported in $2.3 \%$ of cases of patients treated with dabigatran and $2.2 \%$ of patients treated with the standard anticoagulant therapy [13]. In case of apixaban, $0.6 \%$ of "major" bleeding complications (vs. I.8\% in the standard therapy) were reported. Concerning the study endpoint, in apixaban group in $2.3 \%$ VTE recurrence or death due to this disease was reported (vs. 2.7\% in standard therapy) [14].

There are also some pooled analyses of results available $[15,16]$. In the analysis of the results of the phase 3 trials with the use of rivaroxaban (pooled analysis: EINSTEIN DVT and PE), the primary effectiveness endpoint was reported in $2.1 \%$ of patients in the group receiving rivaroxaban and in $2.3 \%$ of patients treated with the standard anticoagulant therapy (major bleedings in $1.0 \%$ and $1.7 \%$ of patients, respectively) [15].

All of the above-mentioned studies show that the safety and effectiveness of new anticoagulants are at least similar to the safety and effectiveness of the current therapy recommended for the treatment and prevention of the recurrence of deep vein thrombosis [1I, 14].

Non-vitamin $\mathrm{K}$ antagonist oral anticoagulants may be used (in accordance with the marketing documents) both in the chronic treatment and in the treatment of the acute phase of thrombosis [7-10]. No need to supervise the treatment, fixed dose and simple use (that does not require the assessment of coagulation parameters) are definitely the advantages of new anticoagulants [17-20]. Another unquestionable advantage is a potentially greater possibility of ensuring proper and stable anticoagulation level with the use of new anticoagulants. The proportion of patients remaining within the therapeutic range of anticoagulation (INR range 2-3) leaves much to be desired even in randomized clinical trials of phase 3 , and in the routine clinical practice it seems to be even lower [II-I4, $2 \mid]$. Therefore, the use of new oral anticoagulants in the treatment, especially in the treatment of patients with an unstable INR range, gives an opportunity to increase the proportion of patients who are properly and effectively treated with an anticoagulant therapy.

Of course, it is necessary to take appropriate safety measures, especially in the scope of the evaluation of renal function as well as current risk of bleeding, just like in any other clinical situation associated with the anticoagulant therapy [7-10, 18-20]. In case of renal function evaluation, one should take into account both baseline parameters of renal function and its possible changes during further treatment $[7-10,20,22,23]$. A correct baseline creatinine clearance does not release 
patients from the obligation to control this parameter regularly (in case of patients without primary renal impairment at least once a year and in case of patients with primary renal impairment — more often; this also applies to situations related with the risk of the sudden deterioration of renal function, e.g. dehydration, infection, fever, especially in the elderly) [7-10].

The simultaneous use of other antithrombotic drugs, including antiplatelet drugs, is a factor indicating a higher risk of bleeding in case of using oral direct coagulation inhibitors. However, it should be emphasized that despite the fact that the combination of new anticoagulants with an antiplatelet therapy, especially with a dual antiplatelet therapy, increases the risk of bleeding, similar observations were also made in relation to the use of warfarin together with the antiplatelet therapy [24-28]. Currently available recommendations, in particular those concerning coronary revascularization or coronary revascularization in patients with atrial fibrillation, contain combination treatment regimens using new direct coagulation inhibitors with an antiplatelet therapy, including dual antiplatelet therapy [22, 29]. Nonetheless, these documents emphasize the possibility of increasing the risk of bleeding complications in case of the combination therapy, suggesting at the same time that the triple anticoagulation therapy with the use of new anticoagulants should be kept to a minimum [22, 29]. The above-mentioned recommendations requiring caution in case of the combination treatment refer in this regard (increased risk of bleeding complications in case of the simultaneous antithrombotic therapy) to the general principles of using antithrombotic therapy included in the previous recommendations [22, 29].

An increasing number of patients treated with anticoagulants or using other types of the antithrombotic therapy and an increasing population of patients with renal impairment and elderly patients requiring an anticoagulant treatment are undoubtedly a significant problem in the routine clinical practice. In this respect one should also take into account the group of patients from the population of so-called fragile patients patients in whom the risk of complications, including bleeding complications, is increased. Summaries of product characteristics of particular drugs also draw attention to patients characterized by an increased risk of bleeding related to age and concomitant diseases (including renal failure) [7-10].

NOAC can be used in a monotherapy (treatment of the acute phase and chronic treatment with the use of the same type of drug) or in combination with other anticoagulants. The possibility of using the so-called single drug approach, i.e. the treatment based on only one type of drug, relates to some molecules from the group of factor Xa inhibitors (rivaroxaban, apixaban) [12, 14].
Currently used anticoagulant therapies involving the use of parenteral, short-acting anticoagulants in the acute phase of thrombosis and then oral drugs can be replaced by using rivaroxaban $(2 \times 15 \mathrm{mg}$ for 21 days and then $I \times 20 \mathrm{mg})$ or apixaban $(2 \times 10 \mathrm{mg}$ for 7 days and then $2 \times 5 \mathrm{mg}$ ) from the beginning of the treatment $[7,9,12,14]$. In case of using drugs from the group of thrombin inhibitors, the registered treatment regimen involves the initial treatment with the use of a short-acting anticoagulant (heparin, low molecular weight heparin) and then (after at least 5 days of the treatment) an oral drug (dabigatran) administered in two daily doses [9].

The use of new anticoagulants is based on the currently available recommendations and previously conducted phase 3 trials, but they do not always take into account the actual range of patients treated in particular centers as part of the routine clinical practice. A certain group of patients was excluded from each of the phase 3 trials during screening, which resulted from the need to meet the criteria adopted in the period of eligibility of patients [ $1 \mathrm{I}-14]$. On the other hand, in spite of meeting the criteria included in the current summaries of product characteristics, in the routine clinical practice we often meet patients whose condition as well as other factors having an impact on the risk of bleeding complications and the risk of recurrence were not fully described both in the previously conducted studies and in the marketing documents.

In view of the above, it is vitally important to conduct studies related to the verification of the effectiveness and safety of drugs used in the routine clinical practice. So far, prospective observational studies of patients treated with new direct coagulation inhibitors are available in the literature concerning atrial fibrillation [30-34]. Such data largely confirm previous observations made on the basis of prospective phase 3 trials suggesting a low proportion of bleeding complications as well as satisfactory outcome in case of drugs to which they related [30-34]. So far, there is no direct prospective comparison between the outcome of the new treatment modalities and the group of patients treated using the treatment method based on drugs from the group of vitamin $\mathrm{K}$ antagonists in patients with deep vein thrombosis among post-marketing prospective clinical trials related to new anticoagulants and so-called routine clinical practice. XALIA (XA inhibition with rivaroxaban for Long-term and Initial Anticoagulation in venous thromboembolism) was the first post-marketing, multicenter study on this matter [35].

The XALIA study was an international, prospective and observational study conducted in hospitals and outpatient health centers in 21 countries. The aim of the study was to assess the effectiveness and safety of 
rivaroxaban which was registered in December 2011 by the European Medicines Agency (EMA) as the first drug from the group of direct coagulation inhibitors for the treatment of deep vein thrombosis as well as for the secondary prevention of the recurrence of venous thromboembolism in adults [35].

The study began in June 2012 and 5142 patients were enrolled in it in the period from June 26, 2012 to March 3I, 20I4. Initially, only the patients with deep vein thrombosis were taken into consideration. After the registration of rivaroxaban for the treatment of pulmonary embolism, the patients with deep vein thrombosis and concomitant pulmonary embolism were also qualified. The XALIA study was non-interventional and the study protocol was approved by EMA. The study was supervised by the scientific steering committee in the scope of the study design, clinical protocol, data verification and result analysis.

Patients who were over 18-years-old with objectively confirmed deep vein thrombosis as well as indications for at least 3-month anticoagulant treatment using rivaroxaban or the standard treatment method based on unfractionated heparin, low molecular weight heparin or fondaparinux and vitamin $\mathrm{K}$ antagonists were eligible to participate in the study. After the registration of rivaroxaban for the treatment of pulmonary embolism, the study protocol was completed, providing for the possibility of enrolling patients with confirmed combined deep vein thrombosis and pulmonary embolism; patients with pulmonary embolism, but without confirmed deep vein thrombosis were excluded from the study. All patients enrolled in the study gave their informed consent for the participation in the study. 6839 patients were evaluated during the screening period, but only 5 I 42 were enrolled - 2I49 patients in the group of standard therapy and 2619 in the group treated with rivaroxaban. The type, dose and duration of antithrombotic (anticoagulant) therapy were determined by the attending physician. Patients who were treated using an anticoagulant therapy without previous parenteral treatment and patients in whom the treatment with rivaroxaban followed the treatment with heparin or fondaparinux no longer than 48 hours were enrolled in the group treated with rivaroxaban (according to the protocol of the EINSTEIN DVT study) [II]. All patients enrolled in the study were followed for at least 12 months (until the end of 12 months from the moment of the enrollment of the last patient). Patient data were collected during the visit enrolling in the study and during visits in the follow-up period (without any interferences in the treatment regimen). In case of patients who were not consulted again for any reason, information concerning their condition and possible adverse events were collected by phone, e-mail or mail after one month from the enrollment in the study at 3-month intervals and during the last visit. Bleeding complications, cases of the recurrence of venous thromboembolism as well as other adverse events were recorded in the medical history during the follow-up period on the basis of the previously prepared questionnaire. According to the study protocol, patients in whom symptoms or signs suggesting the occurrence of any of the safety endpoints (bleeding, adverse events) occurred were referred to or encouraged to visit hospital to have their complaints examined and to obtain final diagnosis. Patients who were initially eligible for the group treated with rivaroxaban, but who finally received treatment using heparin or fondaparinux in the period of at least 2-14 days with or without a simultaneous use of vitamin $\mathrm{K}$ antagonists in the period of $\mathrm{I}-\mathrm{I} 4$ days before switching to rivaroxaban are referred to as "early switchers" (368 patients) — such patients were excluded from the primary safety analysis.

The effectiveness and safety of treatment with the use of each of treatment regimens were evaluated during the analysis of the results. The primary endpoint included the incidence of adverse events connected with the treatment such as: major bleeding complications, recurrence of symptomatic venous thromboembolism and death from any reason. All adverse events connected with the treatment were also recorded. To compare the populations with similar characteristics, in the group and result comparison, the analysis based on the propensity score was used. The recurrence of venous thromboembolism was defined as the re-occurrence of the symptoms of the disease subsequently confirmed by the objective diagnostic tests. Deaths were classified as caused by bleeding complications, venous thromboembolism and other reasons. Secondary endpoints included: the incidence of major cardiovascular events (cardiovascular death, stroke, myocardial infarction, unstable angina, acute coronary syndromes), other symptomatic cardiovascular events (Budd-Chiari syndrome, retinal vein thrombosis, cerebral venous sinus thrombosis, portal vein thrombosis, cathether-related thrombosis, upper limb venous thrombosis - if the primary thrombotic event concerned lower limbs), the necessity of the hospital admission (including hospital stay length assessment), the level of patient satisfaction with the proposed treatment regimen and the adherence to the medical guidelines concerning the VTE therapy as well as the presence of other side effects. The safety analysis concerned the evaluation of primary and secondary endpoints in the group of patients who received at least one dose of the anticoagulant ("safety" population) - the analysis included the results related to 4768 patients: 2619 patients in the rivaroxaban group and 2149 patients in the group 
Table I. Baseline characteristics of the groups [35]

\begin{tabular}{|c|c|c|c|}
\hline & Rivaroxaban & Standard therapy & $\mathbf{p}$ \\
\hline Age & 59.0 years $(45-7 I)$ & 66 years $(47-73)$ & $\mathrm{p}<0.001$ \\
\hline Diagnosis & & & $\mathrm{p}<0.001$ \\
\hline Deep vein thrombosis & $92 \%$ & $88 \%$ & \\
\hline Deep vein thrombosis and pulmonary embolism & $8 \%$ & $12 \%$ & \\
\hline Type of thrombosis & & & 0.0033 \\
\hline Known risk factor (provoked) & $34 \%$ & $38 \%$ & \\
\hline Idiopathic (unprovoked) & $65 \%$ & $61 \%$ & \\
\hline No data & $1 \%$ & $1 \%$ & \\
\hline Active cancer when enrolled into the study & $6 \%$ & $19 \%$ & $p<0.0001$ \\
\hline Previous events of major bleeding & $1 \%$ & $3 \%$ & 0.0002 \\
\hline First creatinine clearance result available & & & $p<0.0001$ \\
\hline$<30 \mathrm{~mL} / \mathrm{min}$ & $1 \%$ & $3 \%$ & \\
\hline$\geq 30<50 \mathrm{~mL} / \mathrm{min}$ & $3 \%$ & $7 \%$ & \\
\hline$\geq 50<80 \mathrm{~mL} / \mathrm{min}$ & $16 \%$ & $19 \%$ & \\
\hline$\geq 80 \mathrm{~mL} / \mathrm{min}$ & $43 \%$ & $37 \%$ & \\
\hline None & $37 \%$ & $34 \%$ & \\
\hline
\end{tabular}

using the standard anticoagulant therapy. The analysis was carried out in the entire study population as well as in 8 homogeneous population subgroups covered by the comparative assessment based on the propensity score implementation.

When comparing baseline characteristics of the groups (related to the use of both treatment methods in the routine clinical practice), it should be noted that patients receiving rivaroxaban in the study populations were generally younger and fewer of them had a known cancer or pulmonary embolism as compared to the group of patients receiving standard anticoagulant therapy (Table I).

In the group receiving rivaroxaban $72 \%$ of patients received it as the only treatment method whereas $28 \%$ of patients were treated with parenteral anticoagulant administered for the maximum period of 2 days before the first dose of rivaroxaban. Therefore, the proportion of patients treated only with rivaroxaban from the beginning of the treatment in the XALIA study significantly exceeded the proportion of patients treated with the use of this kind of the single drug approach in the EINSTEIN DVT study [II]. Among 2I 49 patients treated with the standard anticoagulant regimen, the average time in which INR was maintained within the therapeutic range was $56.2 \%$ of the treatment duration (SD 38.2). The average duration of the follow-up in the group treated with rivaroxaban was 239 days (IQR I54-388) and 284 days ( I I I-408) in the standard anticoagulant therapy group.
As far as the assessment of both groups in terms of safety is concerned, it should be noted that major bleedings were observed less frequently in the group treated with rivaroxaban than in the case of the standard anticoagulant therapy (Table 2). There were no deaths caused by bleeding complications in the group treated with rivaroxaban and two deaths caused by such complications in the group treated with the standard therapy. Documented cases of the recurrence of venous thromboembolism as well as deaths (irrespective of the causes of death) less frequently concerned the group of patients taking rivaroxaban than the group of patients using standard anticoagulant therapy (Table 2).

The annual incidence of major bleeding complications, the recurrence of venous thromboembolism and death (regardless of their cause) concern respectively I. $2 \%, 2.4 \%$ and $0.8 \%$ of the patients treated with rivaroxaban and $3.4 \%, 3.9 \%$ and $6.2 \%$ of the patients treated with the standard anticoagulant therapy. Cancer was the most frequent cause of death in both groups and the mortality rate associated with venous thromboembolism was similar $(0.2 \%)$ in both cohorts.

Due to the above-mentioned baseline differences between these two populations of patients (including lower average age in patients treated with rivaroxaban), both patient groups were compared again after group matching on the basis of the analysis based on the propensity score. The above-mentioned analysis was carried out in order to compare the effectiveness and safety of the treatment in the groups of patients 
Table 2. Results of the treatment of the study populations in the XALIA study [35]

\begin{tabular}{|c|c|c|c|}
\hline & Rivaroxaban & $\begin{array}{l}\text { Standard } \\
\text { anticoagulant } \\
\text { therapy }\end{array}$ & $\begin{array}{l}\text { HR } \\
\text { (hazard risk) }\end{array}$ \\
\hline \multicolumn{4}{|l|}{ Major bleeding complications } \\
\hline Any & $0.7 \%$ & $2.3 \%$ & 0.41 \\
\hline Fatal & 0 & $\begin{array}{c}0.1 \% \\
(2 \text { deaths })\end{array}$ & \\
\hline From the digestive tract & $0.1 \%$ & $0.8 \%$ & \\
\hline Into the central nervous system & $0.2 \%$ & $0.2 \%$ & \\
\hline Any bleeding reported & $11.4 \%$ & $10.1 \%$ & \\
\hline \multicolumn{4}{|l|}{ Effectiveness of the treatment } \\
\hline Recurrence of venous thromboembolism & $1.4 \%$ & $2.6 \%$ & 0.67 \\
\hline \multicolumn{4}{|l|}{ Type of recurrence of venous thromboembolism } \\
\hline Fatal pulmonary embolism & $<0.1 \%(1)$ & $<0.1 \%(1)$ & \\
\hline $\begin{array}{l}\text { Death in case of which pulmonary embolism cannot be excluded } \\
\text { as a cause of death }\end{array}$ & $0.2 \%(4)$ & $0.2 \%(4)$ & \\
\hline Non-fatal pulmonary embolism & $0.6 \%$ & $0.8 \%$ & \\
\hline $\begin{array}{l}\text { Recurrence of deep vein thrombosis with concomitant } \\
\text { pulmonary embolism }\end{array}$ & $<0.1 \%(1)$ & $0.2 \%(4)$ & \\
\hline Recurrence of deep vein thrombosis & $0.5 \%$ & $1.4 \%$ & \\
\hline \multicolumn{4}{|l|}{ Other } \\
\hline Major cardiovascular events & $0.3 \%$ & $0.6 \%$ & 0.64 \\
\hline All-cause mortality & $0.5 \%$ & $4.1 \%$ & 0.26 \\
\hline \multicolumn{4}{|l|}{ Causes of death } \\
\hline \multicolumn{4}{|l|}{ Death caused by venous thromboembolism } \\
\hline Pulmonary embolism & $<0.1 \%(1)$ & $<0.1 \%(1)$ & \\
\hline $\begin{array}{l}\text { Death in case of which pulmonary embolism cannot be excluded } \\
\text { as a cause of death }\end{array}$ & $0.1 \%(3)$ & $0.1 \%(3)$ & \\
\hline Death related to cancer & $0.2 \%$ & $2.8 \%$ & \\
\hline Death from cardiovascular causes & $<0.1 \%(1)$ & $0.5 \%$ & \\
\hline Other & $<0.1(1)$ & $0.6 \%$ & \\
\hline
\end{tabular}

who are as similar as possible in terms of the baseline characteristics.

2505 patients taking rivaroxaban and 2010 patients taking standard anticoagulants were analyzed using propensity score matching. I 4 patients from the group receiving rivaroxaban and 139 patients receiving standard anticoagulant therapy were excluded from the analysis due to different values in the propensity score model. After the exclusion of such patients, the cohorts of patients were similar and there were no statistically significant differences in the analysis in the scope of primary endpoints (including major bleedings, venous thromboembolism recurrence and death from any cause). The incidence of major bleedings in the analysis taking into account matching on the basis of the propensity score was $0.8 \%$ in the group treated with rivaroxban and $2.1 \%$ in the group treated with the standard anticoagulant therapy [propensity score adjusted hazard ratio (HR) $0.77(95 \% \mathrm{Cl}: 0.4-\mathrm{I} .5)$; $p=0.44]$. The incidence of the recurrence of venous thromboembolism was $1.4 \%$ and $2.3 \%$ respectively [propensity score adjusted hazard ratio (HR) 0.91 (95\% Cl: 0.54-I.54); $p=0.074]$. A similar proportion of adverse events was observed in both groups $(36.0 \%$ vs. $37.5 \%$ ) as well as a comparable proportion of major cardiovascular events was noticed. The analysis related to subgroups of patients did not show any significant differences between the patients taking rivaroxaban and using the standard treatment method in the scope of the incidence of major bleedings and the recurrence of venous thromboembolism in relationship to the patient's weight, age, initial presence of cancer and renal function impairment. There were no significant differences in the scope of secondary endpoints either - the incidence of major cardiovascular events and other thromboembolic events in patients treated with 
rivaroxaban or standard anticoagulant therapy did not differ to a statistically significant extent. Similar observations were also made in relationship to differences in the scope of adverse events.

To sum up, the results of the XALIA study confirm the results of previous findings based on the prospective randomized phase 3 studies in the population characterized by the wide range of patients. Such results indicate a high safety and effectiveness of rivaroxaban in the treatment of venous thromboembolism in the routine clinical practice.

As mentioned above, the XALIA study is the first international, multicenter, prospective clinical trial concerning the use of a direct coagulation inhibitor rivaroxaban in the routine clinical practice in patients with deep vein thrombosis. The fact that younger patients who less frequently suffered from pulmonary embolism were enrolled in the group treated with rivaroxaban may reflect the physicians' approach to the use of a new treatment modality at the early stage of their clinical experience with this type of the therapy (the XALIA study began in less than a year after the registration of rivaroxaban for this indication and the amendment of the protocol was introduced after the registration of the indication regarding the treatment of pulmonary embolism by the study drug). However, due to the use of the analysis based on the propensity score, it is possible to compare both groups of patients with similar baseline parameters and only a small group of patients in the group treated with rivaroxaban and vitamin $\mathrm{K}$ oral antagonists was excluded from the analysis. 125 different clinical characteristics, including age, gender, weight, renal impairment or presence of pulmonary embolism, were taken into account in the analysis - the aim of which was to match both study populations to the greatest extent possible [35].

The results of the XALIA study give an answer to a number of important questions related to the use of the new oral anticoagulants, in particular rivaroxaban that was evaluated in the study, in the routine clinical practice. As mentioned above, $72 \%$ of patients in the arm of the study treated with rivaroxaban received the treatment in the form of a monotherapy (single drug approach), which means that rivaroxaban was administered from the beginning of the treatment without a previous anticoagulant therapy using parenteral drugs. The proportion of patients treated only with rivaroxaban was therefore more than twice as high as in the phase 3 EINSTEIN DVT study and the results obtained in this way confirm the effectiveness and safety of this type of the treatment in the routine clinical practice as well $[11,35]$. The incidence of major bleedings in the group of patients taking rivaroxaban was $0.7 \%$ as compared to $0.8 \%$ of patients receiving a standard an- ticoagulant therapy. The proportion of the recurrence of venous thromboembolism was also low and similar to the results obtained in the EINSTEIN DVT study [XALIA - I.4\% vs. EINSTEIN DVT - 2.1\%] [I I, 35].

Taking into account a relatively small number of major bleedings observed in the group treated with rivaroxaban as well as vitamin $\mathrm{K}$ antagonists, it should also be mentioned that there were no cases of fatal bleeding in the population taking rivaroxaban and evaluated in the XALIA study.

The treatment regimen in case of bleeding and its final clinical outcomes are important subjects related to each form of antithrombotic treatment, including both antiplatelet and anticoagulant therapy [22, 36, 37]. Also in the subjects qualified to NOAC treatment, the current beeding risk should be assessed. An important point is also the potential renal function impairment evaluation as well as its further control in the course of NOAC treatment $[19,20,22,36]$. Currently, a registered antidote against new direct inhibitors of anticoagulation is available only for dabigatran, but there are ongoing advanced studies related to obtaining clinical confirmation of the possibility of using an antidote for drugs from the group of factor $X a$ inhibitors, e.g. adnexanet alpha [38-40]. While waiting for the results of this studies, it is worth noting that despite the fact that the availability of antidotes seems to be, by all means, justified, according to the earlier observations in most cases bleeding complications reported during the use of new anticoagulant molecules most likely do not require the use of a specific antidote [22, 36, 39]. The above-mentioned observations were mostly made on the basis of the pharmacological properties of new oral anticoagulants and, above all, their relatively short time of action [6-10]. In most cases, it is sufficient to discontinue medication and replace blood loss (if needed) $[30,31,36,37]$.

The increasing knowledge of physicians regarding new molecules and their use is another factor that seems to be important in terms of the safety of the use of direct coagulation inhibitors. The above observations can also apply, for instance, to post-marketing studies of patients with atrial fibrillation in whom new treatment methods are used. In the XANTUS study, major bleeding was reported in $2.1 \%$ of patients per year and the incidence of stroke was $0.7 \%$ per year [33].

In case of the Post-Marketing Safety Surveillance carried out in the USA on the basis of the data obtained from the medical database of the Department of Defense in the period from January 2013 to October 20 I4, in the population of 27467 patients in whom anticoagulant treatment with rivaroxaban was used for the first time due to atrial fibrillation, major bleeding complications were reported in $2.89 \%$ of patients per year [4I]. 
The effectiveness and safety of rivaroxaban vs. warfarin (I I 4 I I patients) and the effectiveness and safety of apixaban vs. warfarin (4083 patients) were compared in the REVISIT US (Real-world EVIdence on Stroke prevention In patients with aTrial Fibrillation in the United States) study regarding the use of rivaroxaban, apixaban and warfarin in patients with atrial fibrillation in the routine clinical practice on the basis of the analysis based on the propensity score. The use of rivaroxaban was associated with the reduction of intracranial hemorrhage $(0.49 \%$ vs. $0.96 \%$ /year; $\mathrm{HR} 0.53 ; 95 \% \mathrm{Cl}: 0.35-0.79)$ and with a statistically insignificant trend towards the reduction of ischemic stroke $(0.54 \%$ vs. $0.83 \%$ /year; HR 0.71 ; $95 \% \mathrm{Cl}: 0.47-1.07)$ in comparison with warfarin [42].

The need to hospitalize and the length of hospital stay of patients with deep vein thrombosis are important from the clinical and economic point of view. In the XALIA study, in the analyzed population of patients with venous thromboembolism, $28 \%$ of 2619 patient treated with rivaroxaban and $47 \%$ of 2149 of patients treated with a standard anticoagulant therapy were hospitalized ( $29 \%$ and $46 \%$ after taking into account the analysis based on the propensity score). The average length of hospital stay was significantly shorter in the group treated with rivaroxaban (5.0 days $\pm 0.07 \mathrm{SE}$ ) than in the group treated with a standard anticoagulant therapy (7.7 days $\pm 0.04 \mathrm{SE}$ ) [35]. It is undoubtedly an important argument in the economic assessment of the total costs connected with the treatment of venous thromboembolism. Of course, further costs of outpatient treatment, treatment costs, costs of reimbursement and availability of drugs for a big group of patients should also be taken into account.

The results of the phase 3 studies and positive results of observational studies and registries kept in the post-marketing period in relation to new oral anticoagulants used for the treatment of venous thromboembolism are reflected in the currently available indications, including the latest version of the 2016 ACCP consensus [3]. Recommendation no. 2 of this document suggests that dabigatran, rivaroxaban, apixaban or edoxaban should be used as a long-term therapy (first 3 months) in patients with deep vein thrombosis or pulmonary embolism instead of drugs form the group of vitamin 2 antagonists [3]. One should also take into account the level of such recommendations [2B] indicating the clinical suggestion. The above-mentioned level of recommendations arises out of the fact that the majority of phase 3 studies that were conducted met the criteria of non-inferiority studies [43-45]. However, as can be seen on the basis of the presented analysis and the results of the XALIA study, the recommendation of the 2016 ACCP consensus is also confirmed by the results obtained in the routine clinical practice.
The XALIA study concerning the use of rivaroxaban in patients with deep vein thrombosis as well as combined deep vein thromboses and pulmonary embolism in the routine clinical practice confirmed that rivaroxaban as the single drug therapy shows a low proportion of major bleeding complications and the recurrence of venous thromboembolism in the population characterized by a wide range of patients [35]. The use of rivaroxaban reduces the length of hospital stay and costs of hospital treatment as well as it simplifies the therapy of this group of patients in comparison with the standard anticoagulant therapy.

\section{References}

I. Cohen AT, Hamilton M, Mitchell SA et al (2015) Comparison of the Novel Oral Anticoagulants Apixaban, Dabigatran, Edoxaban, and Rivaroxaban in the Initial and Long-Term Treatment and Prevention of Venous Thromboembolism: Systematic Review and Network Meta-Analysis. PLoS One; I0: e0I44856.

2. Hull RD, Gersh MH (2015) The current landscape of treatment options for venous thromboembolism: a focus on novel oral anticoagulants. Curr Med Res Opin; 31: 197-210.

3. Kearon C, Akl EA, Ornelas J et al (2016) Antithrombotic Therapy for VTE Disease: CHEST Guideline and Expert Panel Report. Chest; 149: 315-352.

4. Wells PS, Forgie MA, Rodger MA (2014) Treatment of venous thromboembolism. JAMA; 31 I: 717-728.

5. Vo T, Vazquez S, Rondina MT (2014) Current state of anticoagulants to treat deep venous thrombosis. Curr Cardiol Rep; 16: 463.

6. Kearon C, Akl E, Comerota A (20I2) Antithrombotic Therapy for VTE Disease Antithrombotic Therapy and Prevention of Thrombosis, $9^{\text {th }}$ ed: American College of Chest Physicians Evidence-Based Clinical Practice Guidelines: Antithrombotic Therapy for VTE Disease. Chest; I4I (Suppl): e4I9S-e494S.

7. Charakterystyka produktu leczniczego - Xarelto: www.leki. urpl.gov.pl/files/Xarelto.pdf

8. Charakterystyka produktu leczniczego — Pradaxa: - www.leki.urpl. gov.pl/files/20_Pradaxa.pdf

9. Charakterystyka produktu leczniczego - Eliquis: www.leki.urpl. gov.pl/files/16_Eliquis.pdf

10. Charakterystyka produktu leczniczego — Lixiana: www.ec.europa.eu/ /health/documents/community-register/2015/20150619132091/ /anx_|3209|_pl.pdf

II. The EINSTEIN Investigators, Bauersachs R, Berkowitz SD et al (2010) Oral Rivaroxaban for Symptomatic Venous Thromboembolism. N EngI J Med; 363: 2499-25I0.

12. The EINSTEIN-PE Investigators, Büller HR, Prins $M H$ et al (2012) Oral Rivaroxaban for the Treatment of Symptomatic Pulmonary Embolism. N Engl J Med; 366: I287-1297.

13. Schulman S, Kakkar AK, Goldhaber SZ et al (2014) Treatment of acute venous thromboembolism with dabigatran or warfarin and pooled analysis. Circulation; I 29: 764772.

14. Agnelli G, Buller HR, Cohen A et al (2013) Oral Apixaban for the Treatment of Acute Venous Thromboembolism. N Engl J Med; 369: 799-808.

15. Prins MH, Lensing AW, Bauersachs R et al (2013) Oral rivaroxaban versus standard therapy for the treatment of symp- 
tomatic venous thromboembolism: a pooled analysis of the EINSTEIN-DVT and PE randomized studies. Thromb J; I I: 2 I.

16. Greig SL, McKeage K (2014) Dabigatran etexilate: a review of its use in the treatment of acute venous thromboembolism and prevention of venous thromboembolism recurrence. Drugs; 74: 1785-1800.

17. Marcy TR, Truong T, Rai A (2015) Comparing Direct Oral Anticoagulants and Warfarin for Atrial Fibrillation, Venous Thromboembolism, and Mechanical Heart Valves. Consult Pharm; 30: 644-656.

18. Shamoun F, Obeid H, Ramakrishna H (2015) Novel Anticoagulants in Atrial Fibrillation: Monitoring, Reversal and Perioperative Management. Biomed Res Int; 2015: 42403I.

19. Mekaj YH, Mekaj AY, Duci SB, Miftari El (2015) New oral anticoagulants: their advantages and disadvantages compared with vitamin $\mathrm{K}$ antagonists in the prevention and treatment of patients with thromboembolic events. Ther Clin Risk Manag; 11: 967-977.

20. Gómez-Outes A, Suárez-Gea ML, Lecumberri R, Terleira-Fernández Al, Vargas-Castrillón E (2015) Direct-acting oral anticoagulants: pharmacology, indications, management, and future perspectives. Eur J Haematol; 95: 389-404.

21. Schulman S, Kearon C, Kakkar AK et al (2009) Dabigatran versus warfarin in the treatment of acute venous thromboembolism. N Engl J Med; 36I (Suppl): 23422352.

22. Heidbuchel $H$, Verhamme $P$, Alings $M$ et al (2015) Updated European Heart Rhythm Association Practical Guide on the use of non-vitamin $\mathrm{K}$ antagonist anticoagulants in patients with nonvalvular atrial fibrillation. Europace; 17: 1467-1507.

23. Levy JH, Levi M (2014) New oral anticoagulant-induced bleeding: clinical presentation and management. Clin Lab Med; 34: 575-586.

24. Smith JG, Wieloch M, Koul S et al (2012) Triple antithrombotic therapy following an acute coronary syndrome: prevalence, outcomes and prognostic utility of the HAS-BLED score. EuroIntervention; 8: 672-678.

25. Massie BM, Collins JF, Ammon SE et al (2009) Randomized trial of warfarin, aspirin, and clopidogrel in patients with chronic heart failure: the Warfarin and Antiplatelet Therapy in Chronic Heart Failure (WATCH) trial. Circulation; 119: 1616-1624.

26. Thompson PL, Morton AC (2016) Antithrombotic therapy in patients with combined coronary heart disease and atrial fibrillation. Panminerva Med; 58: 23-33.

27. Thompson PL, Verheugt FW (2014) Managing antithrombotic therapy in patients with both atrial fibrillation andcoronary heart disease. Clin Ther; 36: II76-II8I.

28. Velagapudi P, Turagam MK, Agrawal H, Mittal M, Kocheril AG, Aggarwal K (2014) Antithrombotics in atrial fibrillation and coronary disease. Expert Rev Cardiovasc Ther; 12: 977-986.

29. Roffi M, Patrono C, Collet JP et al (2016) 2015 ESC Guidelines for the management of acute coronary syndromes in patients presenting without persistent ST-segment elevation: Task Force for the Management of Acute Coronary Syndromes in Patients Presenting without Persistent ST-Segment Elevation of the European Society of Cardiology (ESC). Eur Heart J; 37: 267-315.

30. Hecker J, Marten S, Keller L et al (2016) Effectiveness and safety of rivaroxaban therapy in daily-care patients with atrial fibrillation. Results from the Dresden NOAC Registry. Thromb Haemost; II 5: 939-949.
31. Beyer-Westendorf J, Ebertz F, Förster K et al (2015) Effectiveness and safety of dabigatran therapy in daily-care patients with atrial fibrillation. Results from the Dresden NOAC Registry. Thromb Haemost; I 13: 1247-1257.

32. Eikelboom JW, Weitz JI (2015) 'Realworld' use of non-vitamin $\mathrm{K}$ antagonist oral anticoagulants (NOACs): Lessons from the Dresden NOAC Registry. Thromb Haemost; I 13: I 159-I I6I.

33. Camm AJ, Amarenco P, Haas $S$ et al (2016) XANTUS Investigators. XANTUS: a real-world, prospective, observational study of patients treated with rivaroxaban for stroke prevention in atrial fibrillation. Eur Heart J; 37: I I45-। I 53.

34. Peacock $F$ et al (2015) Major bleeding in a post-marketing assessment of 39,052 non-valvular atrial fibrillation patients on rivaroxaban. Abstract P4066. ESC 20I5, 29 August-2 September 2015.

35. Ageno W, Mantovani LG, Haas S et al (2016) Safety and effectiveness of oral rivaroxaban versus standard anticoagulation for the treatment of symptomatic deep-vein thrombosis (XALIA): an international, prospective, non-interventional study. Lancet Haematol; 3: el2-2I.

36. Pernod G, Albaladejo P, Godier A et al (2013) Management of major bleeding complications and emergency surgery in patients on long-term treatment with direct oral anticoagulants, thrombin or factor-Xa inhibitors: proposals of the working group on perioperative haemostasis (GIHP) — March 2013. Arch Cardiovasc Dis; 106: 382-393.

37. Liew A, Eikelboom JW, O'Donnell M, Hart RG (2013) Assessment of anticoagulation intensity and management of bleeding with old and new oral anticoagulants. Can J Cardiol; 29 (Suppl 7): S34-44.

38. Burness CB (2015) Idarucizumab: First Global Approval. Drugs; 75: 2155-2161.

39. Ruff CT, Giugliano RP, Antman EM (2016) Management of Bleeding With Non-Vitamin K Antagonist Oral Anticoagulants in the Era of Specific Reversal Agents. Circulation; 134: 248-26I.

40. Weitz JI, Pollack CV Jr. (20I5) Practical management of bleeding in patients receiving non-vitamin $\mathrm{K}$ antagonist oral anticoagulants. Thromb Haemost; I I4: I I 13- I I 2.

41. Tamayo S, Frank Peacock W, Patel M et al (2015) Characterizing major bleeding in patients with nonvalvular atrial fibrillation: a pharmacovigilance study of 27467 patients taking rivaroxaban Clin Cardiol; 38: 63-68.

42. Coleman $\mathrm{Cl}$, Antz M, Simard E et al (2016) Real-world evidence on stroke prevention in patients with atrial fibrillation in the United States. Presented at: European Cardiac Arrhythmia Society Congress. April 17, Paris, France.

43. Castellucci LA, Cameron C, Le Gal G et al (2014) Clinical and safety outcomes associated with treatment of acute venous thromboembolism: a systematic review and meta-analysis. JAMA; 312: II22-II35.

44. Robertson L, Kesteven P, McCaslin JE (2015) Oral direct thrombin inhibitors or oral factor $\mathrm{Xa}$ inhibitors for the treatment of deep vein thrombosis. Cochrane Database Syst Rev; 6: CDOI0956.

45. Robertson L, Kesteven P, McCaslin JE (2015) Oral direct thrombin inhibitors or oral factor $\mathrm{Xa}$ inhibitors for the treatment of pulmonary embolism. Cochrane Database Syst Rev; 12: CDOI0957. 